\title{
Characterization of 9380 contact binaries from the CRTS Variable Sources Catalogue
}

\author{
F. M. Marsh, ${ }^{1 \star}$ T. A. Prince, ${ }^{2}$ A. A. Mahabal, ${ }^{2}$ E. C. Bellm, ${ }^{2}$ A. J. Drake ${ }^{2}$ \\ and S. G. Djorgovski ${ }^{2}$ \\ ${ }^{1}$ Department of Physics and Astronomy, Pomona College, Claremont, CA 91711, USA \\ ${ }^{2}$ Astronomy Department, California Institute of Technology, Pasadena, CA 91126, USA
}

Accepted 2016 August 19. Received 2016 August 18; in original form 2016 June 20

\begin{abstract}
We construct a sample of 9380 contact binaries (W UMa systems) by using the Catalina Real-Time Transient Survey Variables Sources Catalogue. By measuring brightness change rates, light-curve statistics, and temperatures for this sample, we improve the understanding of contact binary light-curve characteristics, and luminosity variability on decadal time-scales. We show that binaries with convective outer envelopes have a different distribution of lightcurve amplitudes and magnitude differences between eclipse minima than binaries with radiative outer envelopes. We find that more than 2000 binaries exhibit a linear change in mean brightness over the 8-yr timespan of observations with at least $3 \sigma$ significance. We note that 25.9 per cent of binaries with convective outer envelopes exhibit a significant change in brightness, while only 10.5 per cent of radiative binaries exhibit a significant change in brightness. In 205 binaries ( 2.2 per cent), we find that a sinusoid model better describes the luminosity trend within the 8-yr observation timespan. For these binaries, we report the amplitudes and periods (as estimated using observed half-periods) of this sinusoidal brightness variation and discuss possible mechanisms driving the variation.
\end{abstract}

Key words: surveys - binaries: eclipsing - stars: magnetic field.

\section{INTRODUCTION}

Since their first physical characterization in the late 1960s (Lucy 1968a,b), great advancements have been made in the understanding of contact binary (or W UMa) systems. W UMa systems consist of two main-sequence stars that are so close to each other that they exchange mass and energy through a region of physical contact. Analysis of contact binary light curves reveals that the systems exhibit Roche geometry, where a combination of gravitational and rotational forces combine to give contact systems their characteristic 'peanut-like' shapes. These systems are interesting because they provide a unique opportunity to study phenomena such as stellar magnetic activity (Applegate 1992), angular momentum loss (Vilhu \& Rahunen 1981), and thermal relaxation oscillations (Wang 1994). A merger of a contact binary system has been observed in time series photometric data (Tylenda et al. 2011), indicating that these systems can rapidly destabilize. Connections of stellar merger events to red novae and blue stragglers have been brought to light in recent literature (Andronov, Pinsonneault \& Terndrup 2006). Studies of large numbers of contact systems can provide information about

^E-mail: fmarsh@caltech.edu the mechanisms that cause brightness fluctuations and orbital period changes.

Before the 1990s, studies were limited to small numbers of contact systems because of technological constraints. The light curves necessary for the comparison of different contact systems were time consuming to obtain by using single system photoelectric and CCD photometry, because it took an entire night of observing to obtain a light curve of just one contact system. Still, carefully assembled samples of tens of contact systems with common characteristics allowed for comparative studies to be performed (Davidge \& Milone 1984; O'Connell 1951; Qian 2001).

Since the late 1990s, photometric surveys that frequently observe large areas of the sky have come online. Through the careful classification of periodic variable sources in survey data sets, larger and larger samples of contact binary systems have been assembled, making studies of contact binaries as a population possible. Previous surveys have compiled variable star catalogues which include many contact binary systems. Examples of such surveys are the All-Sky Automated Survey (Pojmanski 2000), Robotic Optical Transient Search Experiment (Akerlof et al. 2000), Trans-Atlantic Exoplanet Survey (Devor et al. 2008), Lincoln Near-Earth Asteroid Research program (LINEAR; Palaversa et al. 2013), and Catalina Real-Time Transient Survey (CRTS; Drake et al. 2014a). Researchers have also 
selected pure samples of contact binary systems from large survey data sets for study. Researchers have previously used data from the Optical Gravitational Lensing Experiment (Rucinski 1996), Super Wide Angle Search for Planets (Norton et al. 2011), and CRTS (Drake et al. 2014b) to construct pure contact binary samples for study. Lee (2015) have used this approach to study a pure sample detached eclipsing binaries from the CRTS Variable Sources Catalogue.

In this work, we perform photometric analysis on a large sample of contact binaries from the CRTS Variable Sources Catalogue, by using both Catalina Sky Survey (CSS) and Sloan Digital Sky Survey (SDSS) data. Our aim is to learn how contact binary light-curve morphology changes with photospheric temperature, and to assess the luminosity variability of contact binaries on decadal time-scales. We do not apply physical models to the contact binaries under study, but instead take a phenomenological approach.

In Section 2, we describe the SDSS and CRTS data that we use. In Section 3, we describe how we constructed our sample set from 30743 contact binaries published in Drake et al. (2014a). In Section 4, we describe light-curve statistics calculated for the 13551 binaries that were observed in both SDSS and CRTS data. We describe how the temperature was derived from SDSS colours, including de-reddening. We also describe how some systems were removed from our sample based on SDSS colour and harmonic fit characteristics. In Section 5, we search for variability in the luminosity of the candidates on a decadal time-scale, and apply linear and sinusoidal fits to their mean brightness as a function of time. We also describe how some systems were removed from our sample to remove systematic errors in CRTS photometry. In Section 6, we discuss possible mechanisms responsible for the variability of the candidate systems on a decadal time-scale. In Section 7 , we summarize our findings and mention the types of future observations that can be used to learn more about contact binary systems.

\section{OBSERVATIONS}

In this study, we use data from two separate surveys: (1) we use CRTS data spanning $8 \mathrm{yr}$, which allows us for the variation in the luminosity of each system on a decadal time-scale to be measured, and (2) we use SDSS data which provide multiband photometric measurements taken within the timespan of a few minutes, allowing the temperature of each binary to be measured.

\subsection{CRTS photometry}

The CSS uses three telescopes to survey the sky between declinations of $-75^{\circ}$ and $+65^{\circ}$. Although the CSS was originally designed for the detection of near-Earth Asteroids, the CRTS project aggregates time series photometry for over 500 million stationary 'background' sources (Drake et al. 2009; Djorgovski et al. 2011; Mahabal et al. 2011). CRTS observations are taken in 'white light', i.e. without filters, to maximize survey depth. CRTS can perform photometric measurements on sources with visual magnitudes in the range of $\sim 13-20$. Though we only used $8 \mathrm{yr}$ of data, CRTS continues collecting data to this day.

The CRTS photometry used in this work is publicly accessible through the Catalina Surveys Data Release 2 at crts.caltech.edu.

The number of observations that CSS has collected for the candidate systems that we study ranges from 90 (for the least observed systems) to 540 (for the most observed systems). The median number of CSS observations per candidate system is 336, with a standard deviation of 86 observations. The mean photometric error varies from 0.05 to $0.10 \mathrm{mag}$ for most systems, increasing as a function of CRTS magnitude.

\subsection{SDSS photometry}

The SDSS provides multiband photometry in the $u, g, r, i$, and $z$ bands. Because of its drift-scanning configuration, SDSS is well suited to performing photometry on short-period variable stars $(P<1 \mathrm{~d})$, because all of the bands are exposed within a short time of each other: there is a delay of roughly $5 \mathrm{~min}$ between the exposure of the $g$ and $r$ images (York et al. 2000). We use the SDSS DR10 $(g-r)$ colour to calculate the temperature of the binary systems in this study (Ahn et al. 2014).

\section{CANDIDATE SELECTION}

The initial set of contact binaries from which we derived our sample was selected as described in Drake et al. (2014a). The CRTS photometry for this sample can be accessed publicly at http://nesssi.cacr.caltech.edu/DataRelease/Varcat.html (Drake et al. 2014a).

The Drake et al. (2014a) sample was created by selecting data from the Catalina Surveys Data Release 1, based on the criteria of high Stetson variability index $\left(J_{W S}\right)$ and large standard deviation of brightness measurements $(\sigma)$. Drake performed Lomb-Scargle periodogram analysis (LS; Scargle 1982) on these variables, testing for significant periods. Candidates that passed an LS significance cutoff along with additional data quality cuts were further processed to determine the best period and were then visually inspected. Approximately half of the inspected candidates passed selection and were classified by type (e.g. EW: contact binary, EA: Algol type, RRab: RR Lyrae, etc.) based on period, light-curve morphology, and colour information.

In the Drake et al. (2014a) sample, there are 30743 binaries classified as EW, corresponding to W UMa (contact) binaries. The SDSS photometry was cross-matched to the CRTS photometry by using the Large Survey Database framework (Juric 2012). We searched for SDSS point sources within 3 arcsec of the coordinates of the CRTS candidates, and when a one-to-one match existed, we correlated the photometry and added the candidate to our sample. When a unique match did not exist between the SDSS and CRTS photometry, we did not add the candidate to our sample. We chose the 3 arcsec search radius because CSS pixels subtend 2.5 arcsec. Out of the 30743 sources queried, there were 13551 sources with matching CRTS and SDSS photometry. We will describe the parameters derived for each of these 13551 binary candidates in Sections 4 and 5.

Drake et al. (2014a) have shown that 98.3 per cent of the sources classified as contact binaries in CRTS data are also classified as contact binaries in the analysis of LINEAR data in Palaversa et al. (2013). They have also shown that many of the candidates have SDSS DR10 spectra consistent with known spectral characteristics of contact binaries. Because our contact binary sample is selected from the Drake subset, we expect that it will also have greater than 98 per cent purity.

\section{CALCULATION OF TEMPERATURE AND LIGHT-CURVE STATISTICS}

In this section, we describe the light-curve statistics for the sample. We also describe how the photospheric temperature was calculated from SDSS colours for the sample. Our aim is to discover how light curves vary as a function of photospheric temperature. 


\subsection{Light-curve harmonic fit}

We used the period derived in Drake et al. (2014a) to phase-fold the photometry of every system. For every system, we performed a six harmonic (six sine terms, six cosine terms) fit of the phase-folded photometry, by using gatspy (Vanderplas 2015; VanderPlas \& Ivezic 2015). We chose to use six terms in our model because it provides a balance between flexibility to fit complex light-curve shapes and robustness with sparse data. A model with more than six terms performed better at characterizing well-sampled light curves than models with fewer terms. A six-term model, however, was more likely than a model with fewer terms to produce a non-physical shape when the input light curve was sparsely sampled, or sampled unevenly in phase. At best, the harmonic fit is a good approximation of the true continuous light-curve shape, but sometimes this was not the case. When the range of phases is sparsely or unevenly sampled, the fit is poorly constrained, and often exhibits more than two local maxima and more than two local minima, which is not expected in a physical system with Roche geometry (Lucy 1968b). This also occurs if the variation due to an eclipse has an amplitude comparable to the photometric error.

\subsubsection{Harmonic fit filters}

If the six-term harmonic fit had more than two local maxima and two local minima, we eliminated the system from the sample, because physical eclipsing binary light curves should only have two maxima and two minima (like the light curve in Fig. 3). Other than this criterion, we did not place any constraints on the goodness of fit. This filter eliminated 3062 of the 13551 systems in the original sample (22.6 per cent), thereby reducing the sample to 10488 systems.

We compared the distributions of variability amplitude, and mean CSS magnitude $\left(V_{\mathrm{CSS}}\right)$ for the 3062 systems rejected by the harmonic fit filters to the distributions for the 10488 systems that passed through the filter. In the top panel of Fig. 1, we see that systems with low variability amplitude $(<0.3 \mathrm{mag})$ are more common among the 3062 rejected systems. The harmonic fit filter preferentially rejects bright systems, $\left(V_{\mathrm{CSS}}<13.5\right)$, and faint systems ( $V_{\mathrm{CSS}}$ $>17.0$ ).

A system is likely to be rejected when the variability amplitude is comparable to the photometric errors on individual measurements. Thus, low-amplitude systems, and faint systems (which have large photometric errors) are preferentially rejected by the filter. The preferential rejection of the brightest systems is due to additional light-curve scatter introduced by saturation effects in the CRTS photometry.

It is known that the system geometrical element that most strongly affects contact binary light-curve amplitude is the orbital inclination (Rucinski 1993). It has been shown that orbital inclination can be predicted with a precision of $\pm 3^{\circ}$ based on light-curve data alone (Zeraatgari et al. 2015). Therefore, preferentially rejecting lowamplitude systems will preferentially reject highly inclined systems which only partially eclipse. Since inclination is not a property that is intrinsic to the contact binary system, this is not expected to introduce a physical bias into our sample.

The rejection of faint systems preferentially rejects systems that are intrinsically dim, and distant. The rejection of bright systems preferentially rejects systems that are intrinsically more luminous and close. In the bottom panel of Fig. 1, we see that systems at both extremes in brightness are rejected by the filter, but more faint systems are rejected than bright systems. This is expected
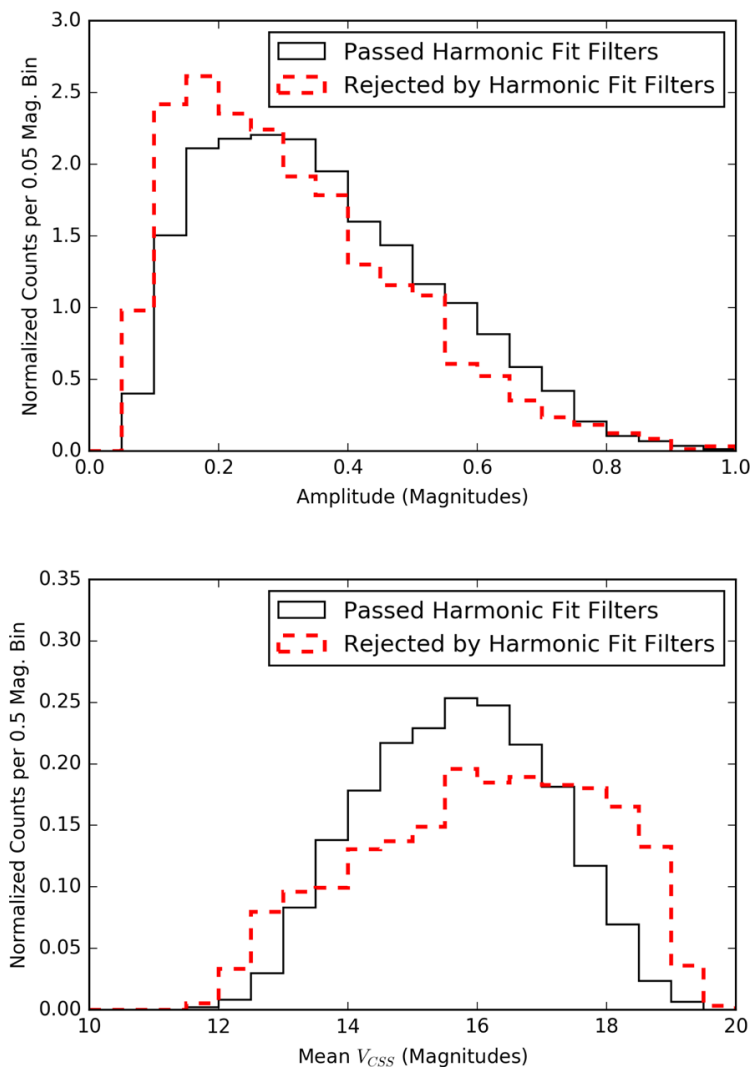

Figure 1. Normalized histograms of variability amplitude (top panel), and mean CSS magnitude $V_{\text {CSS }}$ (bottom panel), for systems retained (black, solid line), and rejected (red, dashed line) by the harmonic fit filters. Systems with low variability amplitude are preferentially rejected by the filters. Systems with mean $V_{\text {CSS }}$ magnitudes of less than 13.5 (the brightest systems) and mean $V_{\mathrm{CSS}}$ of greater than 17 (the faintest systems) are preferentially rejected by the filter.

to bias our sample towards more luminous, hotter, more massive contact binaries with longer orbital periods. We note that the initial sample (like all magnitude-limited samples) is biased towards more luminous contact binaries.

We have not attempted to characterize an unbiased population of contact binaries in this work. A complete and unbiased sample of contact binaries is not a requirement for the analysis performed in this work.

\subsection{Derived light-curve parameters}

For each system in the sample of 10488 , we performed 1000 harmonic fits on Monte Carlo-simulated data sets. Each new simulated photometric measurement was drawn from a normal distribution generated by using the original value of the measurement as the mean, and the reported error as the standard deviation. We sampled these 1000 new harmonic fits at 10000 uniformly spaced points across their phase, and computed the standard deviation of the fits for each point in phase. This was taken to be the standard error at each point in phase on the harmonic fit (Fig. 2). We then retrieved the magnitude and phase of the relative extrema on the harmonic fits, corresponding to the minima and maxima of the eclipses.

The three light-curve parameters (Fig. 3) that we derive in this analysis are amplitude (Amp), magnitude difference between eclipse minima ( $\Delta \mathrm{Min})$, and magnitude difference between 


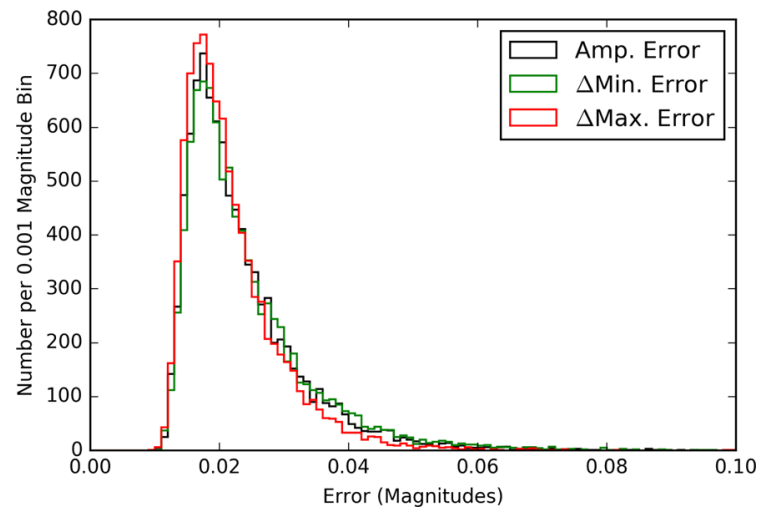

Figure 2. The distribution of errors for the parameters derived from the six-term harmonic fit. A characteristic error for Amp, $\Delta$ Min, and $\Delta$ Max is $0.02 \mathrm{mag}$.

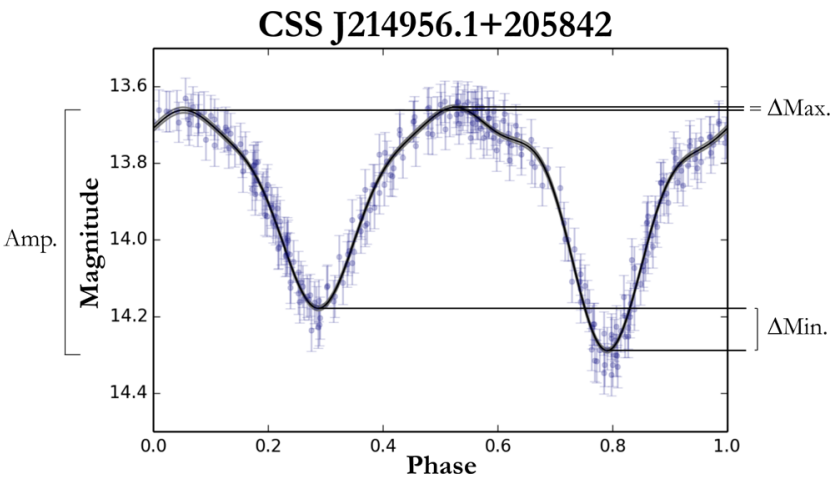

Figure 3. A graphical depiction of the light-curve parameters. Note that $\Delta \mathrm{Max}$ is the very small difference between out-of-eclipse maxima.

out-of-eclipse maxima ( $\Delta$ Max). We define these three parameters to be positive.

\subsection{Effective temperature}

The contact binaries in our sample tended to be further away than those in previous surveys because the contact binaries in our sample were fainter on average than those in previous surveys $\left(\sim 13<V_{\text {CSS }}\right.$ $<20)$ and have a limited range $\left(2 \lesssim M_{\mathrm{V}} \lesssim 7\right)$ of absolute magnitudes (Rucinski \& Duerbeck 1997). Consequently, extra care had to be taken with the de-reddening of the SDSS colours.

The recently released 3D dust map derived from Pan-STARRS1 data by Green et al. (2015) has increased the accuracy with which extinction can be calculated, because the extinction is estimated as a function of direction and distance. If a way exists to roughly determine the distance to a contact binary system by using a method not affected by extinction, we can use the 3D dust map to compute the extinction along the line of sight to the estimated distance, and thereby retrieve a more accurate temperature measurement than is possible with a 2D dust map and Galactic latitude correction.

There is a well-known relationship between the period, colour, and absolute magnitude of contact binary systems. Even without colour information, the absolute magnitude of a contact binary system can be calculated based solely upon its period, with a standard error of 0.3 mag. Rucinski (2006) performed a calibration by using the maximum brightness of 21 contact binary systems with $P<$ $0.56 \mathrm{~d}$ using Hipparcos data and obtained

$M_{\mathrm{V}}=-1.5-12 \log P$, where $P$ is the period in days. This relationship tends to underestimate the absolute magnitude (overestimate the brightness) of systems with $P>0.56 \mathrm{~d}$, by about 0.75 absolute magnitudes. As a result, temperatures will be overestimated for hotter systems. Even with this calibration inaccuracy, the 3D dust map method affords better accuracy than the 2D dust map method, which does not take distance into account at all.

To calculate $\left(V-M_{\mathrm{V}}\right)$, we used equation (1) and the visual magnitude $(V)$ computed from SDSS $g$ and $r$ magnitudes via the empirical relationship $V=g-0.59 \times(g-r)-0.01$ (Jester et al. 2005). The SDSS $g$ and $r$ measurements used to initially estimate the $V$-band magnitude are not corrected for extinction. The difference $\left(V-M_{\mathrm{V}}\right)$ is caused by the dimming due to distance, and the extinction due to reddening. Adopting a ratio of total-to-selective extinction of 3.1, the extinction in the $V$ band, $A_{\mathrm{V}}$, can be expressed as

$A_{\mathrm{V}}=3.1 \times E(B-V)$,

where $E(B-V)$ is the $(B-V)$ reddening. The Green et al. (2015) dust map allows $E(B-V)$ to be calculated as a function of distance modulus, i.e. $E(B-V)=f(D)$. For each system, we can find a distance modulus such that the sum of the distance modulus and $V$ band extinction, $A_{V}$, at that distance modulus is equal to $\left(V-M_{\mathrm{V}}\right)$, because $A_{\mathrm{V}}$ is known as a function of distance modulus. In this way, we can find both the extinction and the distance to the system. We find that 90 per cent of the systems had a $B-V$ reddening, $E(B-V)$, of less than $0.20 \mathrm{mag}$. The median error in $E(B-V)$ was 0.03 mag.

After the best fit of the distance modulus and $E(B-V)$ was calculated, we were able to calculate the extinction in each of the SDSS ugriz bands using the relationships published in Schlafly \& Finkbeiner (2011). We converted $E(B-V)$ to extinctions in each SDSS band. The median extinction in the SDSS $g$ band was 0.11 mag and the median extinction in the SDSS $r$ band was 0.08 mag. Approximately 90 per cent of the candidates had an SDSS $g$ band extinction of less than 0.5 mag. Approximately 90 per cent of the candidates had an SDSS $r$ band extinction of less than 0.3 mag. The median error in the $g$ extinction was 0.10 mag and the median error in the $r$ extinction was 0.07 mag.

We then used an empirical relation (calibrated for main-sequence stars) from Fukugita et al. (2011) to calculate the photospheric temperature of the contact binaries.

$T_{\text {eff }} / 10^{4} \mathrm{~K}=\frac{1.09}{(g-r)+1.47}$.

Temperature calculated from $(g-r)$ colour in this manner carries an uncertainty of $93 \mathrm{~K}$ in the empirically verified range of 3850 $8000 \mathrm{~K}$, which was added in quadrature to the effects of SDSS photometric error and reddening uncertainties. Over 99 per cent of the systems in the sample lie within the empirically verified range.

We note that SDSS provides a temperature measurement at only one phase of the contact binary rotation. The two component stars of a binary system in true contact have temperatures that are within $\sim 100 \mathrm{~K}$ of each other. However, this assumption breaks down for semidetached systems with longer orbital periods.

The uncertainty in the SDSS temperature measurement was computed by adding the provided photometric uncertainties, the reddening uncertainties, and the Fukugita et al. (2011) temperature calibration in quadrature. The resulting mean error in the temperature determination was $324 \mathrm{~K}$, with a standard deviation of $153 \mathrm{~K}$. Systems with lower mean magnitudes tended to have a slightly higher error in the SDSS temperature determination. This is 


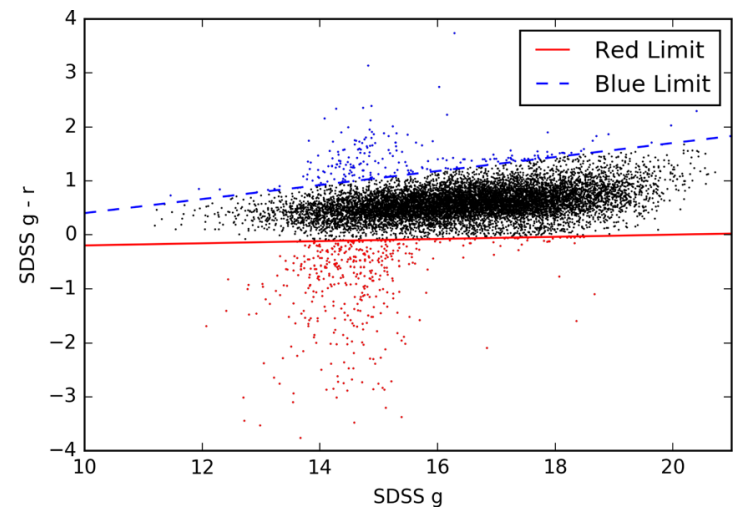

Figure 4. The distribution of the sample in SDSS $(g-r)$ versus $g$ colour space, dashed lines show the red and blue limits imposed upon the data. These limits were imposed to eliminate systems with evidence of saturated SDSS photometry. Points in red and blue show the systems rejected by the respective limits. 661 out of the 10488 systems plotted were eliminated.

because brighter contact binaries are hotter, and hotter binaries tend to have larger temperature errors (because a component of the error is proportional to the temperature).

\subsubsection{Colour limits}

Some of the 10488 system remaining after the harmonic fit filters (Section 4.1.1) exhibited colours that are outside the range expected for contact binary systems. The likely cause of this discrepancy is that much of the SDSS $g$-band photometry is saturated for $g<15$. Systems with saturated photometry were eliminated by choosing a colour cutoff (Fig. 4). We selected both blue and red limits in SDSS $(g-r)$ as a function of SDSS $g$. Candidates were selected such that

$(g-r)>0.02 \times g-0.4($ Red Limit $)$

$(g-r)<0.13 \times g-0.9$ (Blue Limit).

The $(g-r)$ colour derived from SDSS photometry has the largest standard deviation in the SDSS $g$ magnitude range of $\approx 13-15$. Outside of this magnitude range, the systems exhibited a sharp cutoff in $(g-r)$ colour as a function of temperature. On each end of the distribution, a line was fit by eye to the cutoff in the magnitude ranges of 16-20, which was the source of the red and blue limits. Of the 10488 binaries in the sample remaining after the first filter, this eliminated 661, for a new total of 9827 systems.

\subsection{Light-curve shape and temperature}

For each of the 9827 light curves that passed the colour filters (as described in Section 4.3.1), we calculate the amplitude (Amp), difference between eclipse minima $(\Delta \mathrm{Min})$ and the difference between out-of-eclipse maxima $(\Delta \mathrm{Max})$. To generate the plots in this section, we have also filtered out 447 systems that do not pass a criterion described in Section 5.1.1. This is so that the results presented in this section can be compared with those presented in Section 5 (Fig. 5).

The distributions of each of these derived light-curve parameters changes with the effective temperature of the binary system. We find that at certain critical temperatures, many of the characteristics of the systems change.

We discover that the photospheric temperature of $6200 \mathrm{~K}$ divides the binaries in multiple parameter distributions (e.g. Figs 6, 7, and 8),

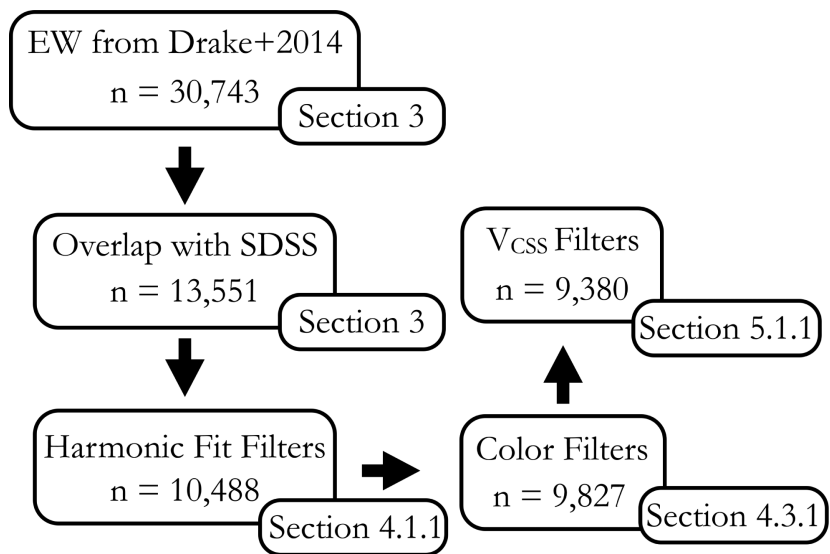

Figure 5. A flowchart describing each of the filters applied to the sample. The type of filter is at the top of each box, while the sample size after applying that filter is at the bottom. The section where each filter is described is at the bottom right of each box.

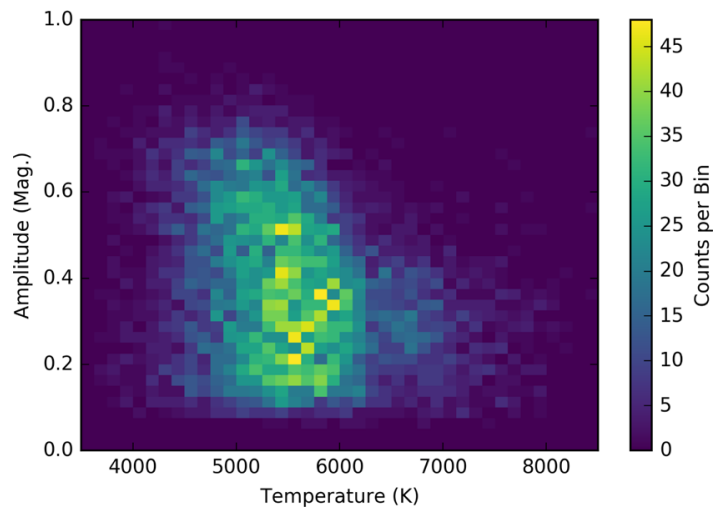

Figure 6. A 2D histogram of amplitude and temperature for the sample. Systems with effective temperatures greater than $6200 \mathrm{~K}$ generally do not have amplitudes greater than $\approx 0.5 \mathrm{mag}$, while systems with effective temperatures lower than $6200 \mathrm{~K}$ do not have amplitudes greater than $\approx 0.8 \mathrm{mag}$. The mean amplitude of the systems cooler than $6200 \mathrm{~K}$ is 0.40 , with a standard deviation of 0.18 . The mean amplitude of the systems hotter than $6200 \mathrm{~K}$ is 0.30 , with a standard deviation of 0.12 .

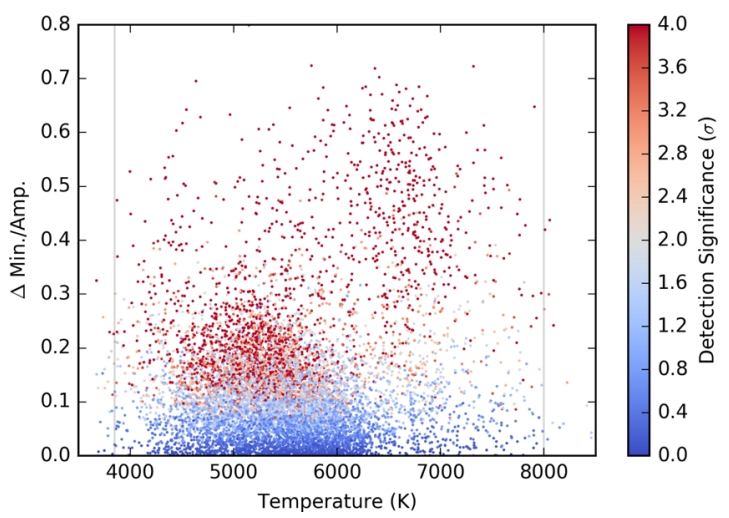

Figure 7. The amplitude-normalized $\Delta$ Min plotted against temperature We normalize by amplitude to control for inclination effects. The colour axis describes the significance of the $\Delta$ Min measurement. $\Delta$ Min measurements that are significant at the greater than $2 \sigma$ level are plotted as red points. Systems with effective temperatures greater than $6200 \mathrm{~K}$ can have much larger $\Delta$ Min than systems cooler than $6200 \mathrm{~K}$. 


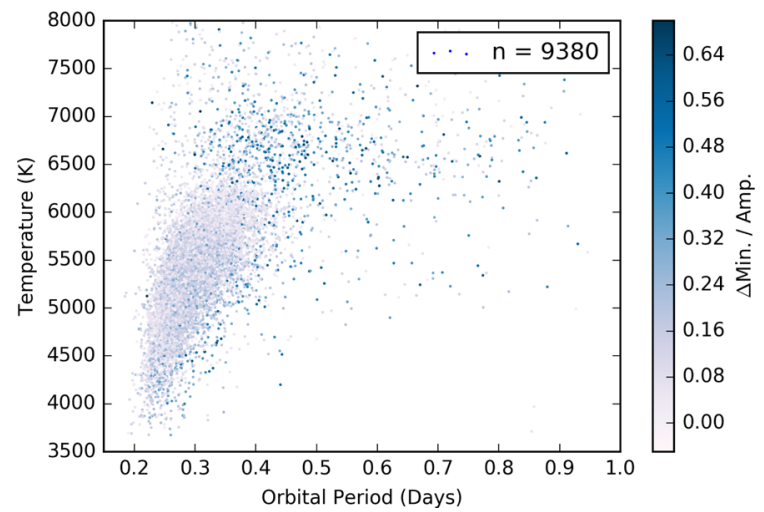

Figure 8. The orbital period and temperature of each contact binary system. $\Delta$ Min normalized to the eclipse amplitude (Amp) is on the colour axis. We have normalized $\Delta \mathrm{Min}$ to Amp. to control for inclination effects. Note that radiative binaries $(T>6200 \mathrm{~K})$ tend to have larger differences between eclipse minima.

and this temperature is important physically. The mode of energy transport at the photosphere changes at the temperature of $6200 \mathrm{~K}$ (p. 212; Kippenhahn, Weigert \& Weiss 1990). Main-sequence stars with temperatures cooler than $6200 \mathrm{~K}$ have a convective outer envelope, while stars hotter than $6200 \mathrm{~K}$ are radiative at the surface. We will refer to the binary systems hotter than $6200 \mathrm{~K}$ as 'radiative' and systems cooler than $6200 \mathrm{~K}$ as 'convective', for short. In our final sample, there are 1381 radiative systems and 7999 convective systems.

Both the system geometry and the inclination of the orbit relative to the earth's line of sight affect the amplitude of a contact binary light curve. We find that the amplitude distribution of the binaries changes dramatically as a function of temperature, convective systems having a wider distribution of amplitudes (Fig. 6).

The temperatures of the two component stars in a contact binary system affect the magnitude difference between eclipse minima in a contact binary light curve. A temperature difference between the primary and secondary components is the cause of a non-zero $\Delta$ Min. We find that the $\Delta$ Min distribution changes significantly as a function of temperature (Fig. 7). Radiative systems typically have larger $\Delta$ Min than convective systems.

Our data show the well-known period-colour relation (Fig. 8) for contact binaries (Rubenstein 2001). We note that the binaries that are cooler than most other binaries with the same orbital period tend to have larger $\Delta \mathrm{Min} / \mathrm{Amp}$ than binaries that are in the middle of the period-colour relation. The component stars of a system with a longer orbital period than that of the majority of binaries with similar temperatures are separated by a larger distance, in accordance with Kepler's Laws. A larger physical separation between the component stars reduces the likelihood that they are in thermal equilibrium. We expect to see a significant $\Delta$ Min in systems where the two components are not in thermal equilibrium.

The difference in out-of-eclipse maxima is affected by an asymmetry in the contact binary system (Fig. 9). A contact binary system exhibiting a significant $\Delta \mathrm{Max}$ is said to exhibit the O'Connell effect (O'Connell 1951). This effect is theorized to be caused by starspots, asymmetric gas impact (Kallrath \& Milone 1999), asymmetric distribution of circum-binary matter (Liu \& Yang 2003), or Coriolis heating (Zhou \& Leung 1990) of the binary's photosphere. We find that 8862 systems in the sample ( 94.5 per cent of the sample) do not have $\Delta$ Max detectable at greater than $2 \sigma$ significance. We observe that 107 systems (1.1 per cent of the sample) exhibit a $\Delta \mathrm{Max}$

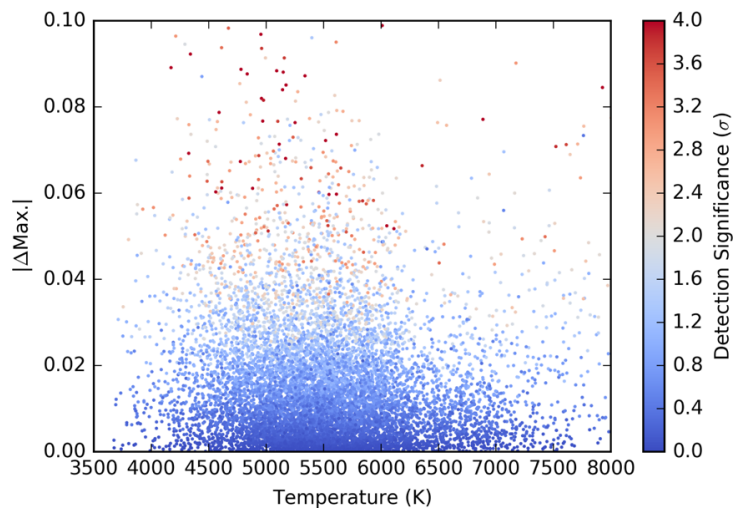

Figure 9. The magnitude difference between eclipse maxima plotted against temperature. The colour axis describes the significance of the $\Delta \mathrm{Max}$ measurement. The vast majority of the systems in the sample do not have a $\Delta$ Max detectable at greater than $2 \sigma$. For light-curve parameters, the typical $2 \sigma$ error is 0.04 mag.

significant at $3 \sigma$. Drake et al. (2014a) have observed the stability of the O'Connell effect over the 8-yr timespan of CRTS observations in this same sample, providing evidence against the theory that starspots are the cause of the effect. This is because starspots can be observed to appear and disappear during the 8-yr timespan of CRTS observations (Fig. 14). It is likely that the cause of O'Connell effect varies from binary to binary. Wilsey \& Beaky (2009) is a review of the possible causes of the O'Connell effect.

\section{SEARCH FOR LUMINOSITY CHANGES ON A DECADAL TIME-SCALE}

\subsection{Linear brightness parameter}

Changes in the mean magnitude of contact binary systems over time-scales of years have previously been attributed to changes in the mean photospheric temperature or mean starspot coverage fraction (Kaszás et al. 1998). To detect luminosity variability on a decadal time-scale, we subtracted the harmonic fit from all of the observations and performed a linear fit on the residuals as a function of time. The six-term harmonic fit effectively eliminated the shortterm variability due to the eclipses. When a single harmonic fit is performed on observations with changing mean brightness over the 8-yr timespan of observations, the residuals are minimized for observations in the middle of the time baseline, but the fit is poor for observations at the beginning and end of the time baseline (Fig. 10). We re-fit 1000 Monte Carlo resamplings of the harmonic fit residuals to perform the linear fit error computation (Fig. 11).

\subsubsection{Mean magnitude filters}

In CRTS data, we find that a systematic error is present in the magnitude derivative calculation. In 2005 and 2006 (the first two years of data in CSDR2), the mean magnitude derivative ( $\left.\dot{V}_{\mathrm{CSS}}\right)$ is positive, instead of being close to zero. In the first two years of photometry, a larger photometric aperture was used for the brighter stars in the sample. Light from additional stars within the aperture was added to the calculated flux. When the aperture size was changed in 2006, the light from the additional stars was lost, resulting in an apparent decrease in brightness. This introduces a systematic error when calculating the trend in brightness over time (Fig. 12). 

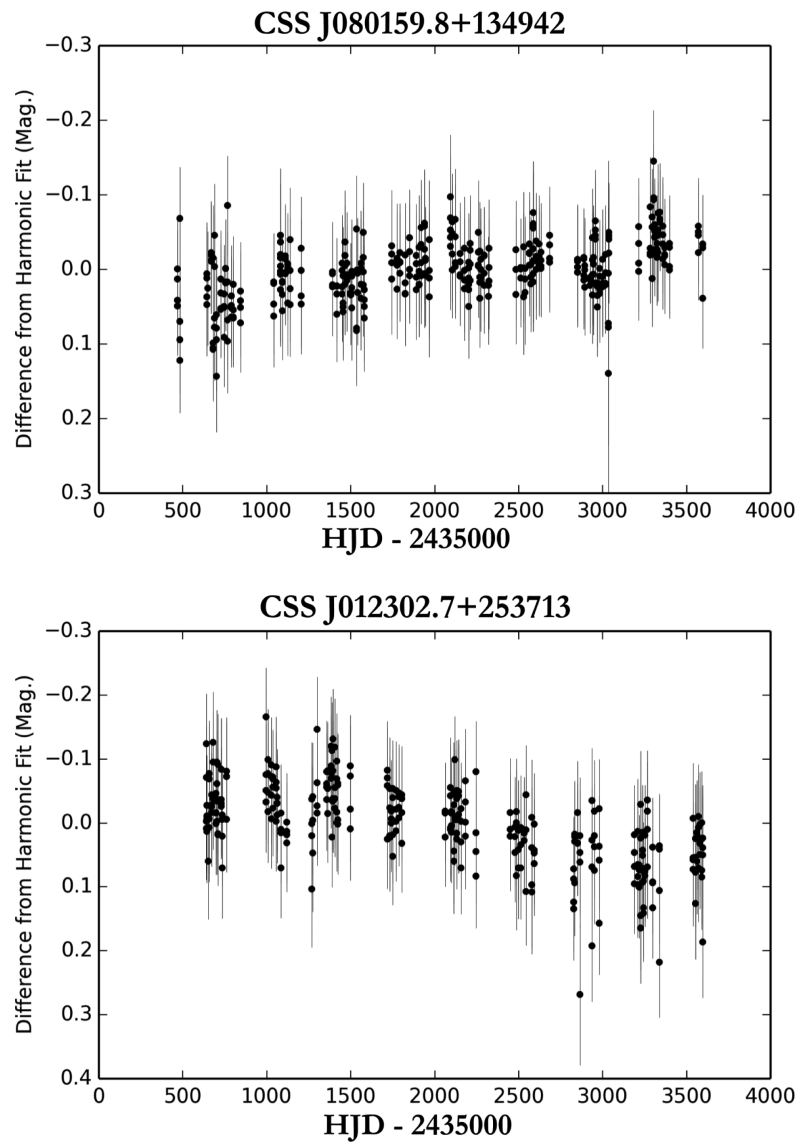

Figure 10. The harmonic fit residuals (and $1 \sigma$ error bars) plotted as a function of time for a system with increasing brightness (top), and for a system with decreasing brightness (bottom) over the 8-yr time baseline of CRTS observations. Top panel: CSS_J080159.8 +134942 , orbital period $=$ $0.35 \mathrm{~d}$. Bottom panel: CSS_J012302.7+253713, orbital period $=0.28 \mathrm{~d}$.

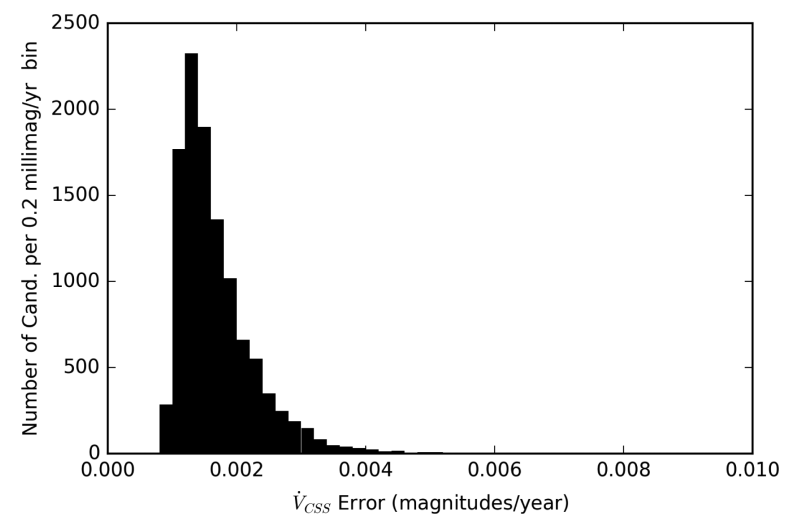

Figure 11. Histogram of the estimated error in the magnitude derivative $\left(\dot{V}_{\mathrm{CSS}}\right)$, if a linear model is assumed. A characteristic error in $\left(\dot{V}_{\mathrm{CSS}}\right)$ is 0.018 mag per year.

To eliminate this systematic error, we rejected all candidates with a mean $V_{\mathrm{CSS}}$ of less than 13.5. This ensures that the distribution of magnitude derivatives is symmetric around zero. This filter eliminated 447 systems from the sample of 9827 remaining after the first two filters, to produce the final number of 9380 systems. All of the analysis in Sections 4 and 5 is based on this final sample of 9380. As was previously mentioned, all of the plots in Section 4.4 were generated using the final sample of 9380 .

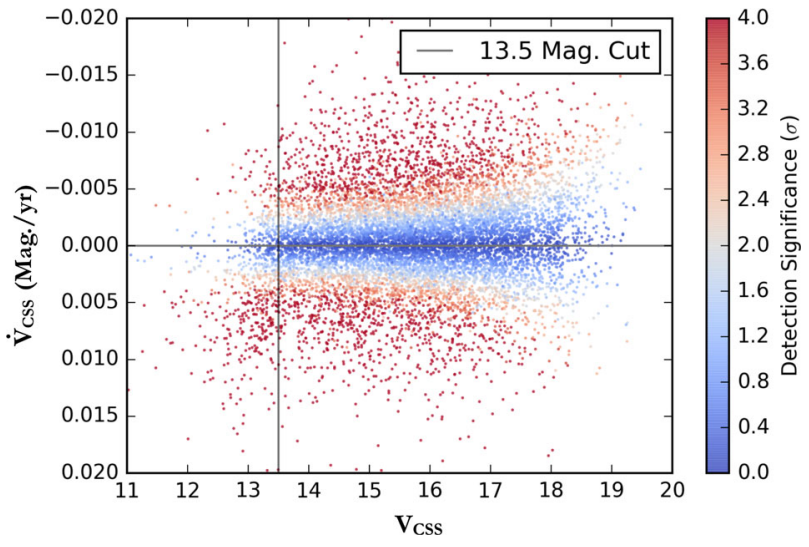

Figure 12. The magnitude derivative ( $\left.\dot{V}_{\mathrm{CSS}}\right)$ of the systems in the sample during the 8-yr observation time period, plotted as a function of CRTS mean magnitude $V_{\text {CSS }}$. The colour axis describes the significance of the $\dot{V}_{\text {CSS }}$ measurement. For systems brighter than magnitude $\approx 13.5$, the distribution of magnitude derivatives is not symmetric around zero, due to changes in the photometric aperture for bright systems. A vertical line is plotted at magnitude 13.5 illustrating the cutoff that we imposed on the data.

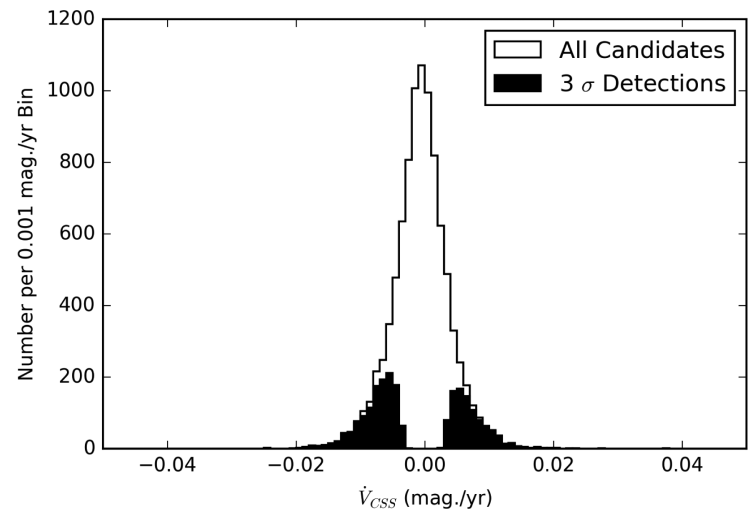

Figure 13. Histogram of the magnitude derivative $\left(\dot{V}_{\mathrm{CSS}}\right)$ in CRTS data. The histogram $3 \sigma$ detections are plotted in black. Of the 9380 systems in the sample, 2219 (23.7 per cent) had linear magnitude changes that were significant at $3 \sigma$.

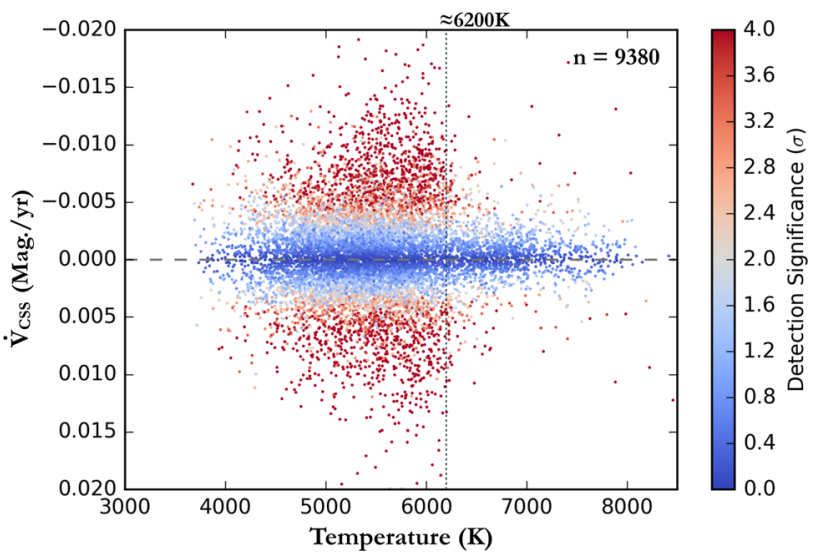

Figure 14. The magnitude derivative $\left(\dot{V}_{\mathrm{CSS}}\right)$ of the systems plotted against their effective temperatures. The colour axis describes the significance of the $\dot{V}_{\text {CSS }}$ measurement. Systems with effective temperatures greater than $\approx 6200 \mathrm{~K}$ do not generally exhibit significant changes in their magnitude. A dashed vertical line indicates the temperature of $6200 \mathrm{~K}$. 


\subsection{Linear brightness parameter and temperature}

As described in Section 5.1, we fit a line to the residuals of the six-term harmonic fit as a function of time, and assessed the significance of the measured slope (the magnitude derivative). For a $3 \sigma$ detection, the probability of the observed data given the null hypothesis of constant magnitude has to be less than $1-0.997=$ 0.003 . We detected $3 \sigma$ significant magnitude derivatives in 2219 systems (23.7 per cent of the sample). The distribution of magnitude derivatives is symmetric about zero, as ensured by the mean magnitude filter described in Section 5.1.1. We discover that some systems have magnitude derivatives as large as 0.02 mag per year (Fig. 13).

The photospheric temperature of $6200 \mathrm{~K}$ separates two distributions of magnitude derivatives. Only 10.5 per cent of the 1381 systems with effective temperatures of greater than $6200 \mathrm{~K}$ exhibit brightness changes significant at $3 \sigma$, while 25.9 per cent of the 7999 systems with effective temperatures of less than $6200 \mathrm{~K}$ exhibit $3 \sigma$ significant brightness changes (Fig. 14).

To determine if the luminosity of systems with a large $\Delta \mathrm{Max}$ (equivalently, systems with a large O'Connell effect) are more likely to vary on a decadal time-scale, we calculated the mean the $\left|\dot{V}_{\mathrm{CSS}}\right|$ distribution as a function of $\Delta$ Max detection significance $(\sigma)$ in six discrete bins (Fig. 15). Since the distribution of $\dot{V}_{\text {CSS }}$ is symmetric about zero, the mean of the absolute value of the distribution is a good way to measure the level of variability. We find that the mean of the $\left|\dot{V}_{\mathrm{CSS}}\right|$ distribution increases smoothly with $\Delta \mathrm{Max}$ significance $(\sigma)$, until the number of systems in each bin is too small to produce reliable statistics. The mean of the $\left|\dot{V}_{\mathrm{CSS}}\right|$ distribution in the $\Delta \operatorname{Max}(\sigma)=3-4$ bin is 1.7 times the mean in the $\Delta \operatorname{Max}(\sigma)=$ $0-1$ bin.

We made an effort to see if the brightness of the systems varied at some orbital phases more than at others. By visually examining the phase-folded light curves of about 20 binaries that exhibited brightness changes significant at $3 \sigma$, it appears that variable binaries vary at all orbital phases. We observed that some of these light curves varied more on one half of the orbital phase than the other. In this work, we will not comment further on the phase variance of the brightness fluctuations.

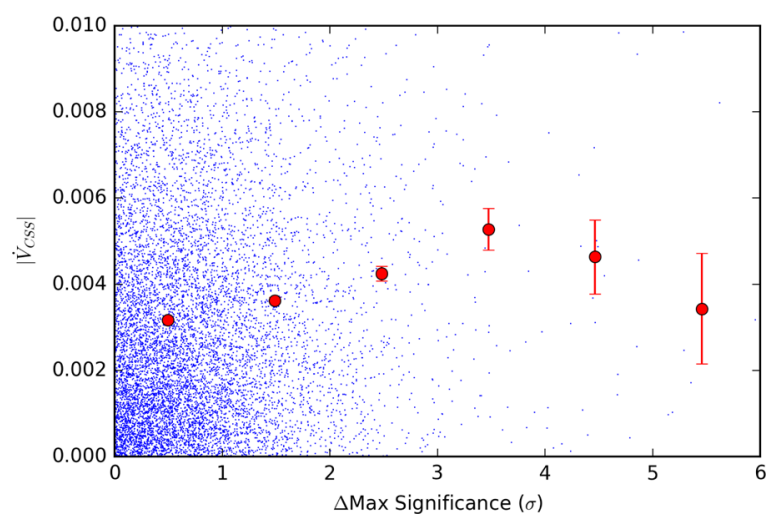

Figure 15. The absolute value of the magnitude derivative $\left|\dot{V}_{\mathrm{CSS}}\right|$ plotted against the significance of the O'Connell effect. The mean and standard error of the mean are computed in $1 \sigma$ wide bins, and graphically represented as red points with error bars. The mean of $\left|\dot{V}_{\mathrm{CSS}}\right|$ in the $\Delta \operatorname{Max}(\sigma)=3-4$ bin is $\approx 0.0053 \mathrm{mag} \mathrm{yr}^{-1}$, while the mean in the $\Delta \operatorname{Max}(\sigma)=0-1$ bin is $\approx 0.0032$ mag $\mathrm{yr}^{-1}$. This difference suggests that systems with a large O'Connell effect are more likely to have a variable luminosity on decadal time-scales.
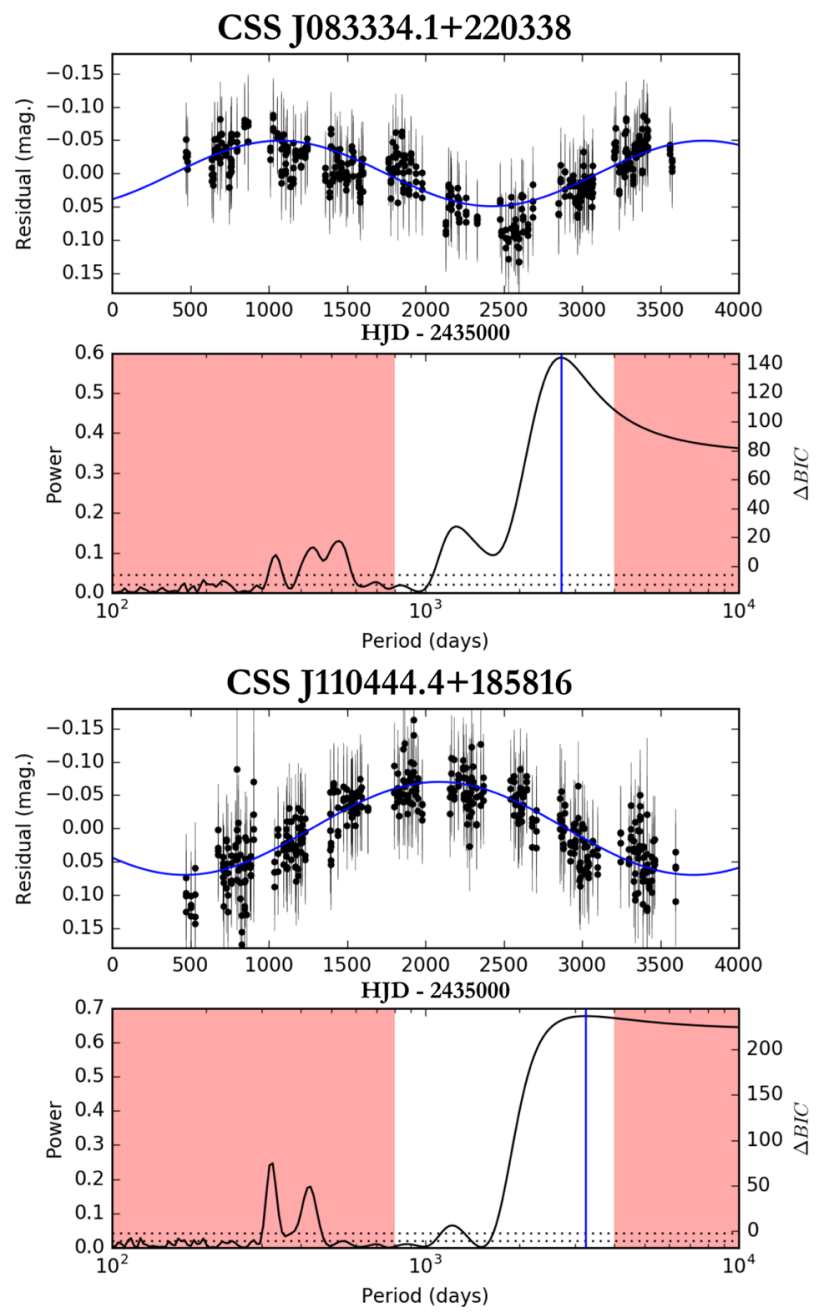

Figure 16. Examples of binaries with evidence of sinusoidal variation in luminosity. This figure shows the luminosity trend of the system after the variation due to eclipsing has been subtracted. The harmonic fit residuals are plotted in the top panel of each plot. $1 \sigma$ error bars are plotted for each harmonic fit residual. A Lomb-Scargle periodogram of the residuals is shown in the bottom panel of each plot. The red regions are periods for which the periodogram is influenced by aliases caused by the sampling pattern of the survey. The two dashed lines near the bottom of the Lomb-Scargle plots are the $1 \sigma$ and $3 \sigma$ significance levels for the periodogram, as computed by Monte Carlo resampling. Top panel: CSS_J110444.4+185816, orbital period $=0.32 \mathrm{~d}$. Bottom panel: CSS_J083334 $1+220338$, orbital period $=$ $0.31 \mathrm{~d}$.

\subsection{Sinusoidal brightness parameters}

We find that for some candidates, the trend in harmonic fit residuals as a function of time is not well described by a linear model. While many systems (like those in Fig. 10) exhibit a monotonic increase or decrease in brightness over the 8-yr CRTS time baseline, many others exhibit one or two points of inflection, where the brightness trend reverses in direction (Fig. 16).

In some cases, the trend in the harmonic fit residuals as a function of time appear to be well described by a sinusoid model. This was especially true for systems whose trends have two points of inflection. In order to quantify the number of systems that exhibited sinusoidal trends in brightness over a decadal time-scale, we performed LS periodogram analysis on all of the harmonic fit residuals as a function of time. 
We cannot say definitively if the luminosity variation of these systems is truly periodic, because in each case, our observations do not capture multiple 'cycles' of the variation. If the variation proves to be truly periodic, we can estimate the period and amplitude of this variation based on observed half-periods. The following analysis assumes that a sinusoid model describes the luminosity variation over time periods longer than the time period of observation. While there are physical reasons to expect that this should be the case (discussed in Section 6), we cannot prove that the luminosity variation is periodic by using CRTS data alone.

The sampling of the CRTS survey and its limited time baseline place limits on the range of luminosity variation periods that we can search. All of the systems are randomly, but uniformly sampled in time, except for gaps that occur every year when the system is only above the horizon in the day and is therefore unobservable by the CSS. This leaves a strong signature in the periodogram of any CSS source (with peaks corresponding to periods of 1 and $2 \mathrm{yr}$ ), and thus periodic signals shorter than roughly two years (we chose the cutoff of $800 \mathrm{~d}$ ) were unable to be measured. The total time baseline of the CRTS survey up to Catalina Surveys Data Release 2 is about 8 yr. Because at least half of a period must be observed to see an inflection point, the 8-yr time baseline sets an upper limit on the longest periods detectable. We chose to filter out all systems with periods of brightness variation that were longer than $11 \mathrm{yr}$.

A system had to pass several tests to be marked as sinusoidally variable.

(1) The period of the long term luminosity variation as determined by an LS periodogram must be greater than $800 \mathrm{~d}$ but less than $4000 \mathrm{~d}$.

(2) The sinusoid model must have a Bayesian information criterion (BIC) of greater than 15, indicating strong evidence for the sinusoid model as compared to the null hypothesis of constant system brightness (Schwarz et al. 1978).

(3) The peak LS power must be five times the $3 \sigma$ power as predicted by 1000 Monte Carlo resamplings - this ensures that only systems with amplitudes large compared to the variation caused by photometric errors alone are considered (VanderPlas, Fouesneau \& Taylor 2014).

If a system passed these three tests, a sinusoid model with a period (in years) and an amplitude (in magnitudes) was fit to the harmonic fit residuals as a function of time (Fig. 16).

205 systems in the 9380 binary sample exhibited oscillating brightness variations as discovered by the LS analysis described in Section 5.3. A sinusoid model was fit to the brightness variations with two parameters: (1) period (in years) and (2) amplitude (in magnitudes). We did not compute errors for the parameters of the sinusoid model. From a visual inspection of the LS periodograms, the lower limit on the error in the period determination can be estimated to be $2-3$ yr. From a visual inspection of the residuals, we can say that the amplitude is only known to a 30 per cent -40 per cent level, at best. These errors, though large, will not affect the very broad conclusions that we are able to draw from this analysis.

From the difference in magnitude between the system at maximum brightness and the system at minimum brightness, we were able to calculate the difference between the maximum and minimum luminosities (Fig. 17). By assuming that the binaries radiate isotropically, we can calculate the fractional luminosity variance:

$$
\frac{|\Delta L|}{L}=\frac{|\Delta F|}{F}=\frac{F_{\max }-F_{\min }}{\bar{F}} .
$$

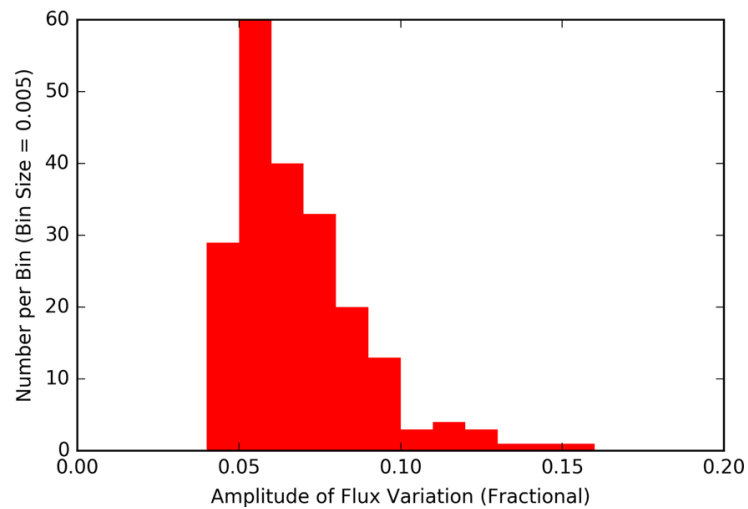

Figure 17. A histogram of the fractional flux (equivalently, the fractional luminosity) variation for the 205 binaries in the sinusoid sample. For a given source, the amplitude errors are at least 30 per cent, as discussed in Section 5.3

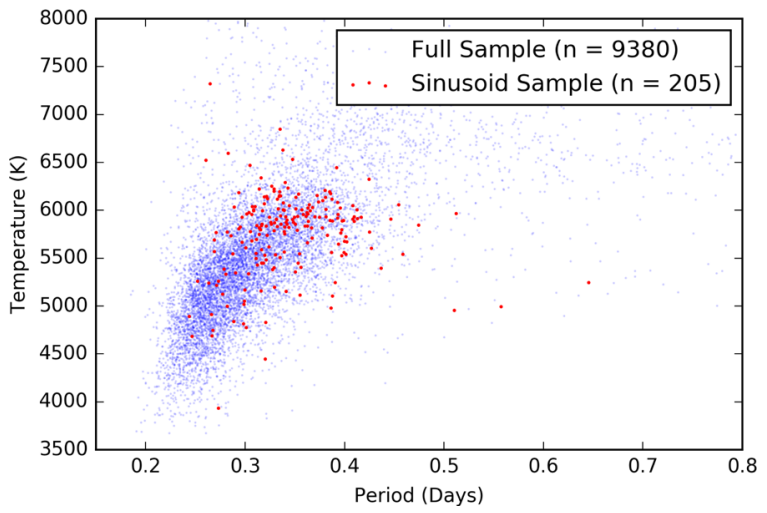

Figure 18. The orbital period and temperature of the 9380 systems in the total sample, and the period and temperature of the 205 systems that exhibit significant sinusoidal variation in their brightness detectable on a decadal time-scale. Systems in the sinusoid sample tend to have higher temperatures and longer periods than systems in the remaining sample.

In general, we observe luminosity variability at the 4 per cent level (the lower limit of detection) to the 16 per cent level. We can state that 2.2 per cent of the contact binaries in the whole sample have luminosities that vary by more than 4 per cent on a decadal time-scale, making this a rare phenomenon. If the variable binaries are assumed to be isotropically radiating perfect blackbodies of constant shape and size, then a fluctuation of the mean photospheric temperature with peak to trough amplitudes ranging from 50 to $150 \mathrm{~K}$ can explain the observed flux variations.

We note that binaries with temperatures ranging from 5600 to $6300 \mathrm{~K}$ are more likely to exhibit periodic luminosity variation (above the 4 per cent level, with a period of less than $11 \mathrm{yr}$ ) than binaries outside that temperature range. Very few binaries hotter than $6300 \mathrm{~K}$ exhibit this luminosity variation, leading us to speculate that convective activity is responsible for driving the luminosity variation (Figs 18 and 19).

In general, systems with photospheric temperatures of less than $\approx 4500 \mathrm{~K}$ did not exhibit significant luminosity variation with periods of less than $11 \mathrm{yr}$. There are two possible explanations: (1) Binaries with temperatures of less than $\approx 4500 \mathrm{~K}$ could have luminosity variation periods that are longer than $11 \mathrm{yr}$ window or, (2) binaries with temperatures of less than $\approx 4500 \mathrm{~K}$ could luminosity variation with amplitudes of less than 4 per cent. 

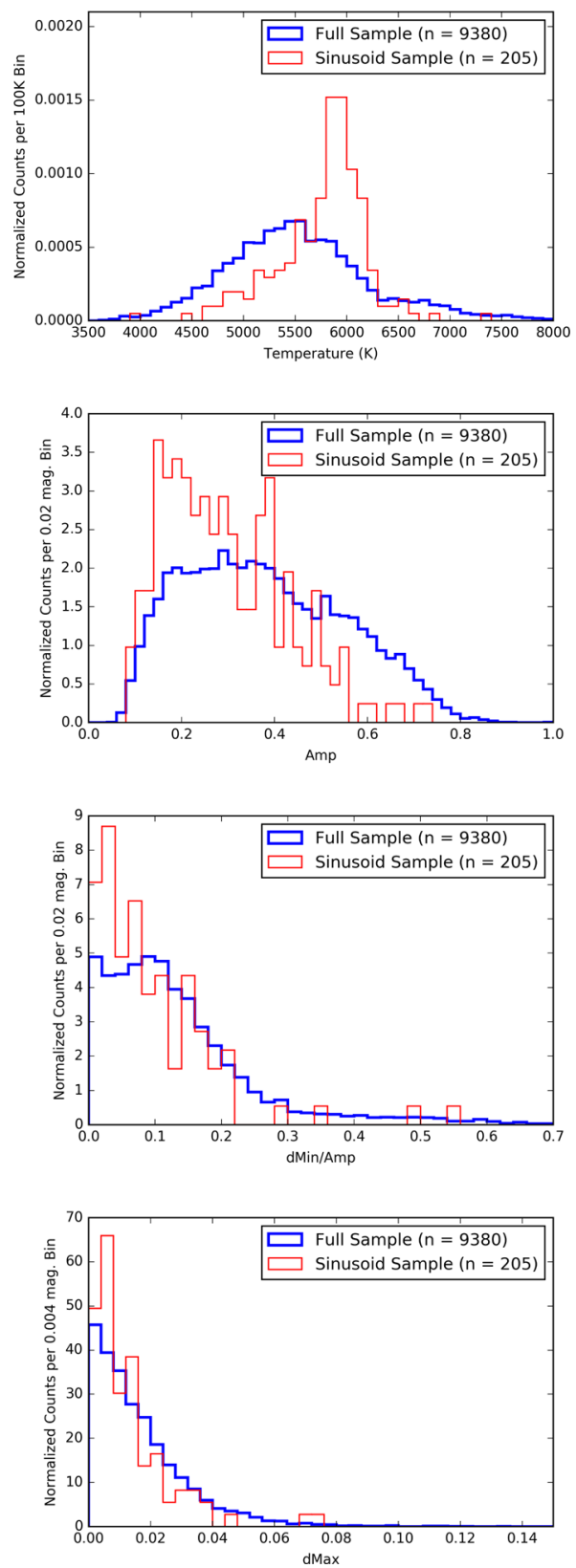

Figure 19. Normalized histograms of the temperature, Amp, dMin, dMax, distribution of systems in the whole sample, and systems in the sinusoid sample showing the overabundance of variable systems in the 5600-6300 K temperature range. Systems in the sinusoid sample have a lower mean Amp, and lower dMin/Amp as compared to systems in the full sample of 9380 .

Binaries in the sinusoid sample have a lower mean light-curve amplitude (Amp) than binaries in the full sample of 9380 (Fig. 19). This could be a selection effect: binaries with low amplitudes were better fit by the six-term sinusoid model, resulting in smaller errors in the fit residuals. This allowed systems with smaller decadal luminosity variations to pass through the filters as described earlier in Section 5.3. The light-curve amplitude of a contact binary is affected strongly by its orbital inclination with respect to the observer, a property not intrinsic to the binary. Thus, it is unlikely that the difference in the light-curve amplitude distribution has a physical cause.

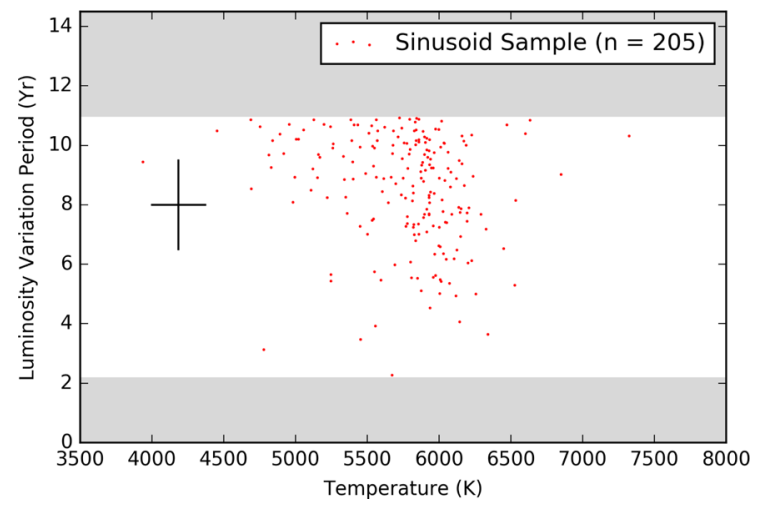

Figure 20. The temperature and luminosity variation period of the 205 systems in the sinusoidal sample. In grey are periods that are not detectable in our analysis. Periods shorter than two years are not detectable due to the annual gaps in sampling due to solar conjunction. A representative error bar is included in black.

We searched for a relationship between the photospheric temperature of the binary and the period of the luminosity variation for the 205 binaries in the sinusoid sample (Fig. 20). Given the large errors, there is no clear relationship in the data, except for the slight suggestion that hotter convective binaries (with temperatures closer to $6200 \mathrm{~K}$ ) exhibit a wide range of luminosity variation periods and are capable of having shorter luminosity variation periods than cooler convective binaries.

We found that four binaries in the sinusoid sample had photospheric temperatures greater than $6200 \mathrm{~K}$ at greater than $1 \sigma$ significance. We examined the SDSS imagery manually for these four binaries. Three out of these four binaries had another star of similar brightness within 5 arcsec. It is possible that the varying brightness of the nearby source is causing the binary to be marked as sinusoidally variable. Another explanation is that an error in the de-reddening procedure could have caused a large error in the temperature measurement - causing an overestimate of the temperature of the star. If the variation is intrinsic to the star and our temperature estimate is accurate, a non-convective mechanism must be causing the luminosity variation in these cases.

\section{DISCUSSION}

In this section, we discuss two possible theories that can explain the decadal luminosity changes observed in 2219 binary systems (23.7 per cent of the 9380), with a particular focus on explaining the sinusoidal luminosity variation detected in 205 binary systems (2.2 per cent of the 9380).

\subsection{The Applegate mechanism}

Applegate (1992) has suggested that orbital period modulations of amplitude $\Delta P / P \approx 10^{-5}$ can be explained by the gravitational coupling of the orbit to variations in the shape of a magnetically active star in the system. Applegate has predicted that the active star be variable at the $\Delta L / L \approx 0.1$ level, the period of this variability matching the period of the orbital period modulation. This luminosity variation should be entirely caused by a temperature variation since large changes in the radius of the star are ruled out by energetics. Under Applegate's model, the period of the luminosity variation is the same as the period of the magnetic activity cycle of the magnetically active star in the system. A detailed discussion of magnetically driven period changes can be found in Lanza (2006). 
We detected luminosity variation at the $0.04 \leq(\Delta L / L) \leq 0.16$ level in 205 contact binaries. While the period of the luminosity variation is poorly constrained in our data, based on observed halfperiods, we can speculate that these 205 binaries have luminosity variation periods ranging from 4 to $11 \mathrm{yr}$, similar to the solar magnetic activity cycle period of $11 \mathrm{yr}$. We estimate that fluctuations of the mean photospheric temperature with amplitudes in the range of 50-150 K can explain the observed flux variations for the binaries in our sample. The vast majority of the variable binaries have photospheric temperatures of less than $\approx 6200 \mathrm{~K}$, indicating that the mode of energy transport near their surfaces is convective. We do not detect decadal variability above the $(\Delta L / L)>0.04$ level in binaries cooler than $\approx 4500 \mathrm{~K}$.

The observed behaviour in these 205 contact binaries matches the predictions made by Applegate's theory. If the Applegate mechanism is indeed responsible for the luminosity variation, this study would add to the evidence for short period magnetic activity cycles on W UMa stars (Kaszás et al. 1998; Shengbang \& Qingyao 2000; Lee et al. 2004; Zhang \& Zhang 2004; Borkovits et al. 2005; Qian et al. 2007; Yang, Qian \& Soonthornthum 2012).

\subsection{Variable starspot coverage}

It is possible that starspots are responsible for the variation in brightness observed by CRTS. Doppler imaging techniques have confirmed the presence of large starspots on the surface of some contact binaries (Barnes et al. 2004). The evolution and migration of starspots on contact binaries has been tracked with doppler imaging (Hendry \& Mochnacki 2000) and more recently, in Kepler data (Tran et al. 2013; Balaji et al. 2015). Starspots are magnetic phenomenon, and so their occurrence is related to the magnetic activity of their host star (Berdyugina 2005). On the sun, the sunspot count varies with the magnetic field strength at the solar surface, which is expected to be true of main-sequence stars in general. As one of the component stars of the contact binary progresses through its magnetic activity cycle, the mean starspot coverage fraction varies, changing the luminosity of the whole system. At this point, we are unsure if the mean photospheric temperature fluctuation of 50 $150 \mathrm{~K}$ is caused by spots, or is truly a uniform global variation. The fact that luminosity variation can be seen to some extent at all the orbital phases of the 20 phase-folded light curves that we examined by eye leads us to believe that the temperature variation cannot be caused by a localized, large spot, but instead must be caused by many smaller spots distributed evenly on the contact binary surface.

Under either the Applegate model, or the variable spot coverage model, the luminosity variation has a period that is the same as the magnetic activity cycle of one of the stars in the system. If the luminosity variation is caused by either model, we can measure the magnetic activity cycle period of large numbers of contact binaries by measuring their luminosity variation period. In contrast, magnetic activity cycle periods are challenging to measure (Vaughan 1983) in single stars.

\section{CONCLUSIONS}

The photospheric temperature of $6200 \mathrm{~K}$ separates binaries into two classes: binaries with convective envelopes $(T<6200 \mathrm{~K})$, and binaries with radiative envelopes $(T>6200 \mathrm{~K})$. We find that radiative binaries generally have larger brightness differences between eclipse minima, indicative of temperature and/or mass differences between the primary and secondary component stars. We find that convective binaries have a larger range of eclipse amplitudes than radiative binaries. We discover $3 \sigma$ significant brightness changes on a decadal time-scale in roughly 20 per cent of the sample. We find that 23.7 per cent of binaries with convective outer envelopes exhibited a significant change in brightness, while only 10.5 per cent of radiative binaries exhibited a significant change in brightness, leading us to believe that the outer convective envelope of the binary is primarily responsible for driving brightness changes. We have detected luminosity variation at the $0.04 \leq(\Delta L / L) \leq 0.16$ level in 205 contact binaries ( 2.2 per cent of the sample). If this luminosity variation proves to be cyclic, we estimate luminosity variation periods ranging from 4 to $11 \mathrm{yr}$. The characteristics of the observed luminosity variation agree well with the predictions of the Applegate Mechanism, in which a luminosity change at the $\Delta L / L \approx 0.1$ level can be explained by the gravitational coupling of the orbit to variations in the shape of a magnetically active star in the system. Alternatively, the luminosity variation can be explained by a variation in the mean starspot coverage fraction of the binary photosphere with the same period as the magnetic activity cycle of the primary. Under either model, the luminosity variation period has the same period as the magnetic activity cycle of one of the stars in the system. If the magnetic interpretation of the brightness variation is correct, measuring the period of decadal luminosity variation will also yield the period of its magnetic activity cycle.

Observations of the 205 sinusoidally variable stars over a time baseline of longer than $8 \mathrm{yr}$ will allow us for a more accurate measurement of the period and amplitude of the luminosity variation on a decadal time-scale. Measurements of the temperature history of variable contact binaries over a multiyear time period will help determine if the Applegate Mechanism is a viable explanation of this phenomenon.

We have included the data used in this study in an online table named Marsh et al 2016 Data.csv, accessible via the online version of this work. This table includes the following for each binary: celestial coordinates, the measured orbital period, the light-curve shape parameters described in Section 4.4 and associated errors, the linear brightness parameter described in Section 5.1 and associated errors, the sinusoidal brightness parameters described in Section 5.3 and associated significance levels, the computed photospheric temperature, and the computed levels of extinction in each SDSS band.

\section{ACKNOWLEDGEMENTS}

This work made use of data products from the CSS survey. The CSS survey is funded by the National Aeronautics and Space Administration under Grant No. NNG05GF22G issued through the Science Mission Directorate Near-Earth Objects Observations Program. The CRTS survey is supported by the US National Science Foundation under grants AST-0909182, AST-1313422, AST-1413600, and AST-1518308.

This work made use of data products from the SDSS-III survey. Funding for SDSS-III has been provided by the Alfred P. Sloan Foundation, the Participating Institutions, the National Science Foundation, and the US Department of Energy Office of Science. The SDSS-III web site is http://www.sdss3.org/.

SDSS-III is managed by the Astrophysical Research Consortium for the Participating Institutions of the SDSS-III Collaboration including the University of Arizona, the Brazilian Participation Group, Brookhaven National Laboratory, Carnegie Mellon University, University of Florida, the French Participation Group, the German Participation Group, Harvard University, the Instituto de Astrofisica de Canarias, the Michigan State/Notre Dame/JINA 
Participation Group, Johns Hopkins University, Lawrence Berkeley National Laboratory, Max Planck Institute for Astrophysics, Max Planck Institute for Extraterrestrial Physics, New Mexico State University, New York University, Ohio State University, Pennsylvania State University, University of Portsmouth, Princeton University, the Spanish Participation Group, University of Tokyo, University of Utah, Vanderbilt University, University of Virginia, University of Washington, and Yale University.

I would like to acknowledge the California Institute of Technology Summer Undergraduate Research Fellowship program for their financial support. I thank an anonymous reviewer for providing comments which greatly improved the paper. I thank James Davenport and Lynne Hillenbrand for their helpful comments, Jake VanderPlas for his help with gatspy, and John McBride for his help preparing the manuscript.

\section{REFERENCES}

Ahn C. P. et al., 2014, ApJS, 211, 17

Akerlof C. et al., 2000, AJ, 119, 1901

Andronov N., Pinsonneault M., Terndrup D., 2006, ApJ, 646, 1160

Applegate J. H., 1992, ApJ, 385, 621

Balaji B., Croll B., Levine A. M., Rappaport S., 2015, MNRAS, 448, 429

Barnes J., Lister T., Hilditch R., Cameron A. C., 2004, MNRAS, 348, 1321

Berdyugina S. V., 2005, Living Rev. Sol. Phys., 2, 8

Borkovits T., Elkhateeb M., Csizmadia S., Nuspl J., Biró I., Hegedüs T., Csorvási R., 2005, A\&A, 441, 1087

Davidge T., Milone E., 1984, ApJS, 55, 571

Devor J., Charbonneau D., O’Donovan F. T., Mandushev G., Torres G., 2008, AJ, 135, 850

Djorgovski S. et al., 2011, in Mihara T., Serino M., eds, The First Year of MAXI: Monitoring Variable X-ray Sources. Special Publ. IPCR-127, p. 263

Drake A. et al., 2009, ApJ, 696, 870

Drake A. et al., 2014a, ApJS, 213, 9

Drake A. et al., 2014b, ApJ, 790, 157

Fukugita M., Yasuda N., Doi M., Gunn J. E., York D. G., 2011, AJ, 141, 47

Green G. M. et al., 2015, ApJ, 810, 25

Hendry P. D., Mochnacki S. W., 2000, ApJ, 531, 467

Jester S. et al., 2005, AJ, 130, 873

Juric M., 2012, Astrophysics Source Code Library, record ascl: 1209.003

Kallrath J., Milone E., 1999, Springer-Velag. New York

Kaszás G., Vinkó J., Szatmáry K., Hegedus T., Gal J., Kiss L., Borkovits T., 1998, A\&A, 331, 231

Kippenhahn R., Weigert A., Weiss A., 1990, Stellar Structure and Evolution. Springer-Verlag, Berlin

Lanza A. F., 2006, MNRAS, 369, 1773

Lee J. W., Kim C.-H., Han W., Kim H.-I., Koch R. H., 2004, MNRAS, 352, 1041

Lee C.-H., 2015, MNRAS, 454, 2946

Liu Q.-Y., Yang Y.-L., 2003, Chin. J. Astron. Astrophys., 3, 142

Lucy L., 1968a, ApJ, 151, 1123

Lucy L., 1968b, ApJ, 153, 877

Mahabal A. et al., 2011, Bull. Astron. Soc. India, 39, 387

Norton A. J. et al., 2011, A\&A, 528, A90
O’Connell D., 1951, Publ. Riverview Coll. Obs., 2, 85

Palaversa L. et al., 2013, AJ, 146, 101

Pojmanski G., 2000, Acta Astron., 50, 177

Qian S., 2001, MNRAS, 328, 635

Qian S.-B., Yuan J.-Z., Soonthornthum B., Zhu L.-Y., He J.-J., Yang Y.-G., 2007, ApJ, 671, 811

Rubenstein E. P., 2001, AJ, 121, 3219

Rucinski S., 1993, PASP, 105, 1433

Rucinski S. M., 1996, AJ, 113, 1112

Rucinski S. M., 2006, MNRAS, 368, 1319

Rucinski S. M., Duerbeck H. W., 1997, in Perryman M. A. C., Bernacca P. L., Battrick B., eds, ESA SP-402: Absolute Magnitude Calibration for the W UMa-type Contact Binary Stars. ESA, Noordwijk, p. 457

Scargle J. D., 1982, ApJ, 263, 835

Schlafly E. F., Finkbeiner D. P., 2011, ApJ, 737, 103

Schwarz G. et al., 1978, Ann. Stat., 6, 461

Shengbang Q., Qingyao L., 2000, Ap\&SS, 271, 331

Tran K., Levine A., Rappaport S., Borkovits T., Csizmadia S., Kalomeni B., 2013, ApJ, 774, 81

Tylenda R. et al., 2011, A\&A, 528, A114

Vanderplas J., 2015, gatspy: General tools for Astronomical Time Series in Python. Available at: http://dx.doi.org/10.5281/zenodo.14833

VanderPlas J. T., Ivezic Ž., 2015, ApJ, 812, 18

VanderPlas J., Fouesneau M., Taylor J., 2014, Astrophysics Source Code Library, record ascl:1407.018

Vaughan A., 1983, in Stenflo J. O., ed., Solar and Stellar Magnetic Fields: Origins and Coronal Effects. Reidel, Dordrecht, p. 113

Vilhu O., Rahunen T., 1981, in Sugimoto D., Lamb D. Q., Schramm D. N., eds, Proc. IAU Symp. 93, Fundamental Problems in the Theory of Stellar Evolution. Kluwer, Dordrecht, p. 181

Wang J.-M., 1994, ApJ, 434, 277

Wilsey N. J., Beaky M. M., 2009, Society for Astronomical. Sciences Annual Symposium, 28, 107

Yang Y.-G., Qian S.-B., Soonthornthum B., 2012, AJ, 143, 122

York D. G. et al., 2000, AJ, 120, 1579

Zeraatgari F., Abedi A., Farshad M., Ebadian M., Riazi N., 2015, Contrib. Astron. Obs. Skalnaté Pleso, 45, 5

Zhang X., Zhang R., 2004, MNRAS, 347, 307

Zhou D.-Q., Leung K.-C., 1990, ApJ, 355, 271

\section{SUPPORTING INFORMATION}

Supplementary data are available at MNRAS online.

\section{Marshetal2016Data.csv}

Please note: Oxford University Press is not responsible for the content or functionality of any supporting materials supplied by the authors. Any queries (other than missing material) should be directed to the corresponding author for the article.

This paper has been typeset from a $\mathrm{T}_{\mathrm{E}} \mathrm{X} / \mathrm{L} \mathrm{T} \mathrm{E} \mathrm{X}$ file prepared by the author. 